\title{
Simplified TeV leptophilic dark matter in light of DAMPE data
}

\author{
Guang Hua Duan, ${ }^{a, b, c}$ Lei Feng, ${ }^{d}$ Fei Wang, ${ }^{e}$ Lei Wu, ${ }^{a}$ Jin Min Yang ${ }^{b, c, f}$ and \\ Rui Zheng ${ }^{g}$ \\ ${ }^{a}$ Department of Physics and Institute of Theoretical Physics, Nanjing Normal University, \\ Nanjing, Jiangsu 210023, China \\ ${ }^{b}$ CAS Key Laboratory of Theoretical Physics, Institute of Theoretical Physics, \\ Chinese Academy of Sciences, \\ Beijing 100190, China \\ ${ }^{c}$ School of Physical Sciences, University of Chinese Academy of Sciences, \\ Beijing 100049, China \\ ${ }^{d}$ Key Laboratory of Dark Matter and Space Astronomy, Purple Mountain Observatory, \\ Chinese Academy of Sciences, \\ Nanjing 210008, China \\ e School of Physics, Zhengzhou University, \\ Zhengzhou 450000, P. R. China \\ ${ }^{f}$ Department of Physics, Tohoku University, \\ Sendai 980-8578, Japan \\ ${ }^{g}$ Department of Physics, University of California, \\ Davis, CA 95616, U.S.A. \\ E-mail: ghduan@itp.ac.cn, fenglei@pmo.ac.cn, feiwang@zzu.edu.cn, \\ leiwu@njnu.edu.cn, jmyang@itp.ac.cn, ruizh@ucdavis.edu
}

ABSTRACT: Using a simplified framework, we attempt to explain the recent DAMPE cosmic $e^{+}+e^{-}$flux excess by leptophilic Dirac fermion dark matter (LDM). The scalar $\left(\Phi_{0}\right)$ and vector $\left(\Phi_{1}\right)$ mediator fields connecting LDM and Standard Model particles are discussed. We find that the couplings $P \otimes S, P \otimes P, V \otimes A$ and $V \otimes V$ can produce the right bump in $e^{+}+e^{-}$flux for a DM mass around $1.5 \mathrm{TeV}$ with a natural thermal annihilation crosssection $\left\langle\sigma v>\sim 3 \times 10^{-26} \mathrm{~cm}^{3} / \mathrm{s}\right.$ today. Among them, $V \otimes V$ coupling is tightly constrained by PandaX-II data (although LDM-nucleus scattering appears at one-loop level) and the surviving samples appear in the resonant region, $m_{\Phi_{1}} \simeq 2 m_{\chi}$. We also study the related collider signatures, such as dilepton production $p p \rightarrow \Phi_{1} \rightarrow \ell^{+} \ell^{-}$, and muon $g-2$ anomaly. Finally, we present a possible $\mathrm{U}(1)_{X}$ realization for such leptophilic dark matter.

KeYWORDS: Phenomenological Models

ARXiv EPrint: 1711.11012 


\section{Contents}

1 Introduction 1

2 Simplified leptophilic dark matter 2

3 Numerical results and discussions 4

4 An $\mathrm{U}(1)_{X}$ realization $\quad 7$

$\begin{array}{llr}5 & \text { Conclusion } & 9\end{array}$

\section{Introduction}

The existence of cold dark matter (CDM) has been confirmed by astrophysical experiments, which provides a natural way to account for many properties of galaxies on large scales. However, the nature of CDM has remained elusive. Among various hypotheses for CDM, the paradigm of weakly interacting massive particles (WIMPs) is one of the most attractive candidates. So far, the WIMP dark matter has undergone very close and effective experimental scrutiny, such as direct detections by measuring the nuclear recoil imparted by the scattering of a DM and collider searches for mono- $X$ signatures.

Besides these, indirect detections via observing high energy gamma-rays, cosmicrays and neutrinos may also shed light on the properties of DM. In past years, several DM satellite experiments, such as AMS-02, PAMELA, HEAT and Fermi-LAT, have been launched and reported some intriguing DM evidences. The DArk Matter Particle Explorer (DAMPE) is a new cosmic ray detector [1,2], which has great energy resolution (better than 1.5\%@TeV for electrons and gamma rays) and good hadron rejection power (higher than $10^{5}$ ). Very recently, DAMPE released their first results about cosmic-ray $e^{+}+e^{-}$ flux up to $5 \mathrm{TeV}$ [3]. A sharp peak at $\sim 1.4 \mathrm{TeV}$ was reported in DAMPE data, which implies the existence of a nearby monoenergetic electron sources because of the cooling process of high energy cosmic-ray electrons $[4,5]$. No associated excess in the anti-proton flux has been observed. Both astrophysical sources (e.g., pulsars) and DM interpretations are discussed in ref. [4]. It is found that DM should annihilate to $e^{ \pm}$or $\left\{e^{ \pm}, \mu^{ \pm}, \tau^{ \pm}\right\}$with 1:1:1 and the mass of DM particle is about $1.5 \mathrm{TeV}$ if the nearby DM sub-halo located at $0.1 \sim 0.3 \mathrm{kpc}$ away from the solar system [4]. Several leptophilic DM model have been proposed to explain this excess $[6,7]$.

In this work, we attempt to explain this tentative cosmic-ray eletron+positron excess by using a simplified framework, in which the DM sector has no direct couplings to quarks, only couples with leptons mediated by a scalar or vector field. Such a leptophilic DM can satisfy the measured relic density at tree level and may accommodate the null results from direct detections by inducing interactions between dark matter and quarks at the loop level. Many studies have been devoted into the idea that DM does not interact with quarks at 


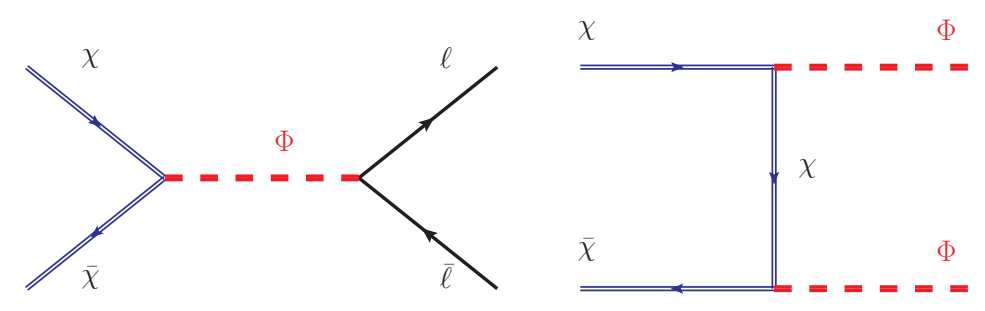

Figure 1. Feynman diagrams for LDM annihilation.

the tree level. Most of these analyses assume an interaction between DM and leptons to be flavor blind [8, 8-39], while a few other studies assume gauged flavor interactions [33, 4048]. The leptophilic DM framework allows for a more general analysis of interactions that involve only DM and leptons at the tree level. It permits different coupling strengths between lepton flavors, off-diagonal flavor couplings, and lepton-flavor violation. ${ }^{1}$

The structure of this paper is organized as follows. In section 2, we introduce the effective lagrangian for leptophilic DM and loop induced LDM-hadron interactions. In section 3, we present our numerical results for the DAMPE excess and discuss the related collider signatures. In section 4 , we give a possible realization of leptophilic DM in U(1) extensions. Finally, we draw our conclusions in section 5 .

\section{Simplified leptophilic dark matter}

The main goal of our study is a model independent analysis of leptophilic Dirac fermion $\mathrm{DM}(\chi)$ for the DAMPE excess. We parameterize the relevant DM-lepton interactions as

$$
\mathcal{L} \ni \Phi_{i} \bar{\chi} \Gamma_{\chi} \chi+\Phi_{i} \bar{\ell} \Gamma_{\ell} \ell
$$

where $\Phi_{i}$ is a mediator field with $i=0,1$ corresponding to spin- 0 and spin- 1 boson respectively. We assume that $\Phi_{i}$ only couples with leptons $e, \mu, \tau$ in our calculations. Then, the Lorentz structures of $\Gamma_{\chi, \ell}$ are scalar (S), pseudo-scalar $(\mathrm{P})$, vector $(\mathrm{V})$ and axial-vector $(\mathrm{A})$ interactions given by

$$
\begin{array}{clc}
\text { scalar-type: } & \Gamma_{\chi}=g_{\chi}^{S}+i g_{\chi}^{P} \gamma_{5}, & \Gamma_{\ell}=g_{\ell}^{S}+i g_{\ell}^{P} \gamma_{5}, \\
\text { vector-type: } & \Gamma_{\chi}^{\mu}=\left(g_{\chi}^{V}+g_{\chi}^{A} \gamma_{5}\right) \gamma^{\mu}, & \Gamma_{\ell \mu}=\left(g_{\ell}^{V}+g_{\ell}^{A} \gamma_{5}\right) \gamma_{\mu},
\end{array}
$$

where $g_{\chi}$ and $g_{\ell}$ are the coupling strengthes of the mediator to DM and SM leptons, respectively.

In our framework, the dominant LDM annihilation channels are

$$
\chi \bar{\chi} \rightarrow \ell \bar{\ell}, \Phi \Phi
$$

with the corresponding Feynman diagrams in figure 1. For a pair of LDM, the CP value of the system is given by $(-1)^{S+1}$. Due to the $\mathrm{CP}$ and total angular momentum conservation,

\footnotetext{
${ }^{1}$ For a review of flavored dark matter, see ref. [49] and the references therein.
} 


\begin{tabular}{|cccc|}
\hline$\Gamma_{\chi} \otimes \Gamma_{\ell}$ & $\sigma v(\chi \chi \rightarrow \ell \bar{\ell})$ & \multicolumn{2}{c|}{$\sigma(\chi N \rightarrow \chi N) /\left(\frac{\alpha_{e m} Z}{\pi m_{\Phi_{i}}^{2}}\right)^{2}$} \\
\hline$S \otimes S$ & $p$-wave & $\alpha_{\text {em }}^{2}$ & {$[2$-loop] } \\
$S \otimes P$ & $p$-wave & - & \\
$P \otimes S$ & $s$-wave & $\alpha_{\text {em }}^{2} v^{2}$ & {$[2$-loop] } \\
$P \otimes P$ & $s$-wave & - & \\
$V \otimes V$ & $s$-wave & 1 & {$[1$-loop] } \\
$V \otimes A$ & $s$-wave & - & \\
$A \otimes V$ & $p$-wave & $v^{2}$ & {$[1$-loop] } \\
$A \otimes A$ & $p$-wave & - & \\
\hline
\end{tabular}

Table 1. Dominant contribution to LDM annihilation cross section and scattering cross section suppression by small parameters for loop induced DM-nucleon scattering for eight Lorentz structures. Here $v$ is the DM velocity.
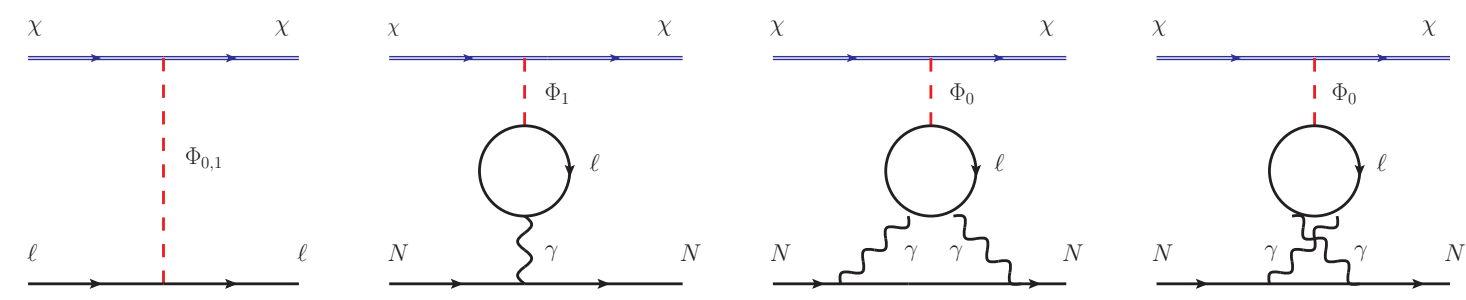

Figure 2. Feynman diagrams for LDM scattering with electron and nucleus.

the quantum states of $|\bar{\chi} \chi\rangle$ are ${ }^{3} P$ and ${ }^{1} S$ for the scalar and pseudoscalar mediators, while the corresponding states for vector and axial-vector mediators are ${ }^{3} S$ and ${ }^{1} P$, respectively. Then, one can estimate the dominant contributions of LDM annihilation cross section, as shown in table 1 . It should be noted that the coupling $A \otimes A$ can produce the $s$-wave contribution, however, which is highly suppressed by mass ratio $m_{\ell}^{2} / m_{\Phi}^{2}$.

Since the LDM only interacts with leptons, it can produce the signal by scattering with electron of atom at tree level or with nucleus at loop level in DM direct detection experiments, as shown in figure 2. The velocity of DM particles near the Earth is of the same order as the orbital velocity of the Sun, $v \sim 0.001 \mathrm{c}$. So the recoil momenta is of order a few $\mathrm{MeV}$, which is much smaller than our mediator mass. Then, we can integrate out heavy mediator fields and obtain the effective operators:

$$
\mathcal{L}_{\text {eff }}=\frac{1}{\Lambda^{2}}\left(\bar{\chi} \Gamma_{\chi} \chi\right)\left(\bar{\ell} \Gamma_{\ell} \ell\right),
$$

where $\Lambda=m_{\Phi} / \sqrt{g_{\chi} g_{\ell}}$ is the cut-off scale for the effective field theory description. With this setup, one can calculate DM-electron scattering cross section at tree level:

$$
\begin{aligned}
\sigma_{\chi e}^{\Phi_{0}} & =\frac{m_{e}^{2} g_{\chi}^{2} g_{\ell}^{2}}{m_{\Phi_{0}}^{4}}\left\{\left(g_{\chi}^{S} g_{e}^{S}\right)^{2}+\left[\left(g_{\chi}^{S} g_{e}^{P}\right)^{2}+\left(g_{\chi}^{P} g_{e}^{S}\right)^{2} \frac{m_{e}^{2}}{m_{\chi}^{2}}\right] \frac{v^{2}}{2}+\frac{\left(g_{\chi}^{P} g_{e}^{P}\right)^{2}}{3} \frac{m_{e}^{2}}{m_{\chi}^{2}} v^{4}\right\}, \\
\sigma_{\chi e}^{\Phi_{1}} & =\frac{m_{e}^{2} g_{\chi}^{2} g_{\ell}^{2}}{m_{\Phi_{1}}^{4}}\left\{\left(g_{\chi}^{V} g_{e}^{V}\right)^{2}+3\left(g_{\chi}^{A} g_{e}^{A}\right)^{2}+\left[\left(g_{\chi}^{V} g_{e}^{A}\right)^{2}+3\left(g_{\chi}^{A} g_{e}^{V}\right)^{2}\right] \frac{v^{2}}{2}\right\} .
\end{aligned}
$$


We can find that DM-electron scattering cross sections for $S \otimes P, P \otimes S$ and $P \otimes P$ couplings are suppressed by both small mass ratio $m_{e} / m_{\chi}$ and low velocity $v \sim 10^{-3}$, while for $V \otimes A$ and $A \otimes V$ couplings the cross sections are only suppressed by velocity. All of them are below the current sensitivity of DM-electron scattering experiments.

The loop induced DM-nucleus scattering cross sections for spin-1/0 mediator at oneloop/two-loop level in leading log approximation [50] are given by:

$$
\begin{aligned}
& \sigma_{\chi N}^{\Phi_{0}}=\frac{\mu_{N}^{2}}{\pi}\left(\frac{\alpha_{e m} Z}{\pi m_{\Phi_{0}}^{2}}\right)^{2}\left(\frac{\alpha_{e m} Z}{\pi}\right)^{2}\left(\frac{\pi^{2}}{12}\right)^{2}\left(\frac{\mu_{N} v}{m_{\ell}}\right)^{2}\left[2\left(g_{\chi}^{S} g_{\ell}^{S}\right)^{2}+\frac{4}{3}\left(g_{\chi}^{P} g_{\ell}^{S}\right)^{2} v^{2} \frac{\mu_{N}^{2}}{m_{N}^{2}}\right] \\
& \sigma_{\chi N}^{\Phi_{1}}=\frac{\mu_{N}^{2}}{9 \pi}\left(\frac{\alpha_{e m} Z}{\pi m_{\Phi_{1}}^{2}}\right)^{2}\left[\sum_{\ell=e, \mu, \tau} \log \left(\frac{m_{\ell}^{2}}{\mu^{2}}\right)\right]^{2}\left[\left(g_{\chi}^{V} g_{\ell}^{V}\right)^{2}+\left(g_{\chi}^{A} g_{\ell}^{V}\right)^{2} v^{2}\left(1+\frac{1}{2} \frac{\mu_{N}^{2}}{m_{N}^{2}}\right)\right]
\end{aligned}
$$

where $m_{N}$ and $Z$ are the nucleus's mass and charge respectively, and $\mu_{N}=\frac{m_{\chi} m_{N}}{m_{\chi}+m_{N}}$ is the reduced mass of DM-nucleus system. The above two-loop result of $\sigma_{\chi N}^{\Phi_{1}}$ is obtained by using operator product expansion in heavy lepton approximation. We set the renormalization scale $\mu=m_{\Phi}$ and both nuclear form factors $F(q)$ for $\Phi_{1}$ and $\tilde{F}(q)$ for $\Phi_{0}$ to unity for simplicity. According to eq. (2.7) and (2.8), we present the scattering cross section suppression by small parameters for loop induced DM-nucleon scattering for eight Lorentz structures in table. 1. It can be seen that the DM-nucleus scattering cross sections for $P \otimes S$ and $A \otimes V$ couplings are suppressed by $v^{2}$, as comparison with $S \otimes S$ and $V \otimes V$ couplings.

\section{Numerical results and discussions}

According to the analysis of ref. [4], the excess of $e^{+}+e^{-}$flux in DAMPE can be interpreted by a DM particle with the mass about $1.5 \mathrm{TeV}$ if the nearby $\mathrm{DM}$ sub-halo locates at $0.1 \sim 0.3 \mathrm{kpc}$ away form the solar system. We fit the AMS-02 and DAMPE data assuming the DM annihilate into leptons with the branching ratio $e: \mu: \tau=1: 1: 1$. Such a condition can evade the constraints from CMB and the diffuse gamma rays from dwarf spheroidal galaxies (dSphs) [4]. In the fitting, we used numerical codes are GALPROP [51] and DRAGON [52] to calculate the propagation of CR electrons/positrons in the galaxy. We use the analytical solution presented in ref. [53] to calculate the propagation of nearby CR electrons. In the first step, we use the LikeDM package [54] to calculate the likelihood (or $\chi^{2}$ ) and fit the AMS-02 and DAMPE data with power-lower background and extra astronomy contribution (see [55] for more details). Then we add the contribution of local $\mathrm{DM}$ halos directly as the local CR source only contributes the region around $1.5 \mathrm{TeV}$. The fitting result is shown in figure 3, in which the mass of DM particles is assumed as $1.5 \mathrm{TeV}$ with the annihilation cross section $\langle\sigma v\rangle \simeq 3 \times 10^{-26} \mathrm{~cm}^{3} \mathrm{~s}^{-1}$ and the mass of nearby subhalo is $1 \times 10^{8} \mathrm{~m}_{\odot}$ with a distance $0.1 \mathrm{kpc}$ away from the solar system.

In order to satisfy DM annihilation cross section, $\langle\sigma v\rangle \simeq 3 \times 10^{-26} \mathrm{~cm}^{3} \mathrm{~s}^{-1}$, required by DAMPE data, we focus on $P \otimes S, P \otimes P, V \otimes A$ and $V \otimes V$ couplings which can produce $s$-wave contributions in our following study. In the following calculations, we assume a universal coupling of the mediator and three generation leptons, $g_{\ell}=g_{e}=$ 


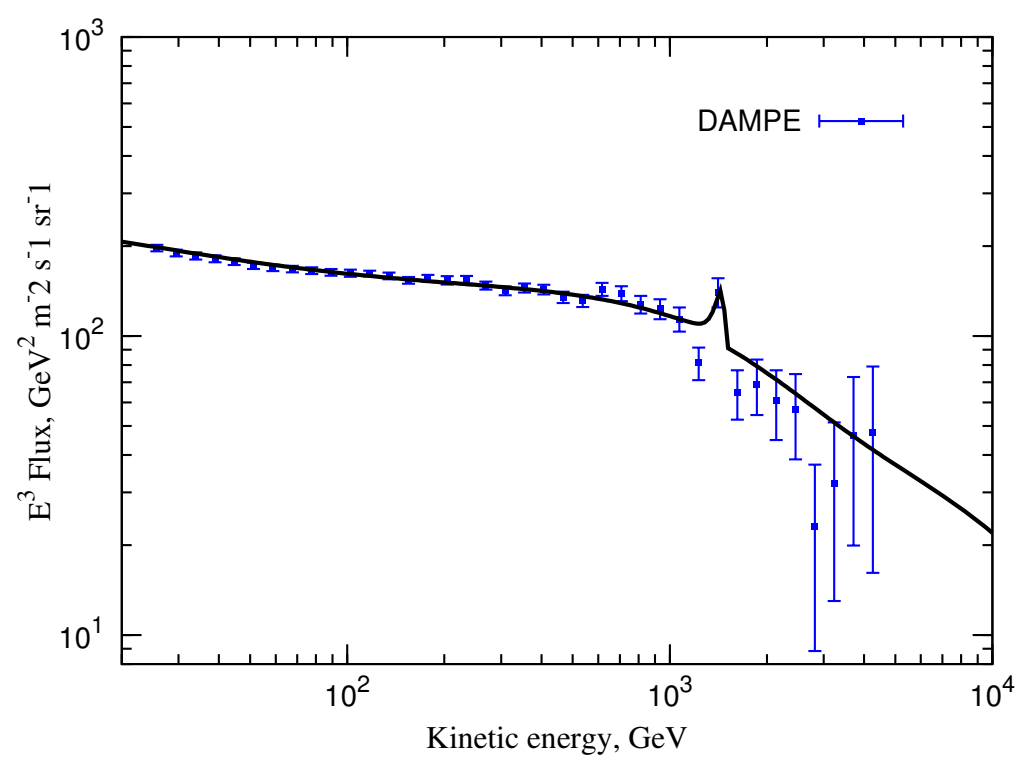

Figure 3. Total $e^{+}+e^{-}$flux of $1.5 \mathrm{TeV}$ DM that annihilates into leptons with the branching ratio $e: \mu: \tau=1: 1: 1$ for fitting AMS-02 AND DAMPE data. The mass of nearby subhalo is assumed as $1 \times 10^{8} \mathrm{~m}_{\odot}$ with a distance $0.1 \mathrm{kpc}$ away from the solar system.

$g_{\mu}=g_{\tau}$. We implement our leptophilic DM model by using FeynRules [56] and evaluate the DM relic density and annihilation cross section with MicrOMEGAs [57]. Since the mediators can induce the process $e^{+} e^{-} \rightarrow \ell^{+} \ell^{-}$, they are strongly constrained by LEP measurements of four-lepton contact interactions [58] and di-lepton resonance searches in $e^{+} e^{-} \rightarrow \ell^{+} \ell^{-} \gamma$ [59]. According the analysis in ref. [60], one can derive the following bounds of the coupling and mass of mediators $\Phi_{0,1}$ at $90 \%$ C.L.,

$$
\begin{aligned}
& g_{\ell}^{V} / m_{\Phi_{1}}< \begin{cases}2.0 \times 10^{-4} \mathrm{GeV}^{-1}, & m_{Z^{\prime}}>200 \mathrm{GeV} \\
6.9 \times 10^{-4} \mathrm{GeV}^{-1}, & 100 \mathrm{GeV}<m_{Z^{\prime}}<200 \mathrm{GeV}\end{cases} \\
& g_{\ell}^{A} / m_{\Phi_{1}}< \begin{cases}2.4 \times 10^{-4} \mathrm{GeV}^{-1}, & m_{Z^{\prime}}>200 \mathrm{GeV} \\
6.9 \times 10^{-4} \mathrm{GeV}^{-1}, & 100 \mathrm{GeV}<m_{Z^{\prime}}<200 \mathrm{GeV}\end{cases} \\
& g_{\ell}^{S, P} / m_{\Phi_{0}}< \begin{cases}2.7 \times 10^{-4} \mathrm{GeV}^{-1}, & m_{Z^{\prime}}>200 \mathrm{GeV} \\
7.3 \times 10^{-4} \mathrm{GeV}^{-1}, & 100 \mathrm{GeV}<m_{Z^{\prime}}<200 \mathrm{GeV}\end{cases}
\end{aligned}
$$

In figure 4 we project the samples satisfying the requirements of DM relic density within $2 \sigma$ range of Planck observed value, LEP bound and the DAMPE excess on the plane of $g_{\chi}$ versus $m_{\Phi}$ for different values of $g_{\ell}$. All samples are required to produce averaged annihilation cross-section $\langle\sigma v\rangle$ today within $(2-4) \times 10^{-26} \mathrm{~cm}^{3} / \mathrm{s}$. When the mass of DM is close to $m_{\Phi} / 2, \mathrm{DM}$ annihilation cross section will be enhanced by resonance effect. In order to satisfy the DM relic density requirement, the couplings $g_{\chi}^{V}$ and $g_{\ell}^{V}$ have to become small, which will suppress the DM-nucleus scattering cross section so that the PandaX-II bound can be evaded [61]. For $P \otimes S$ coupling, the DM-nucleus scattering cross section is highly reduced due to two-loop suppression, while for $P \otimes P$ and $V \otimes A$ couplings, 

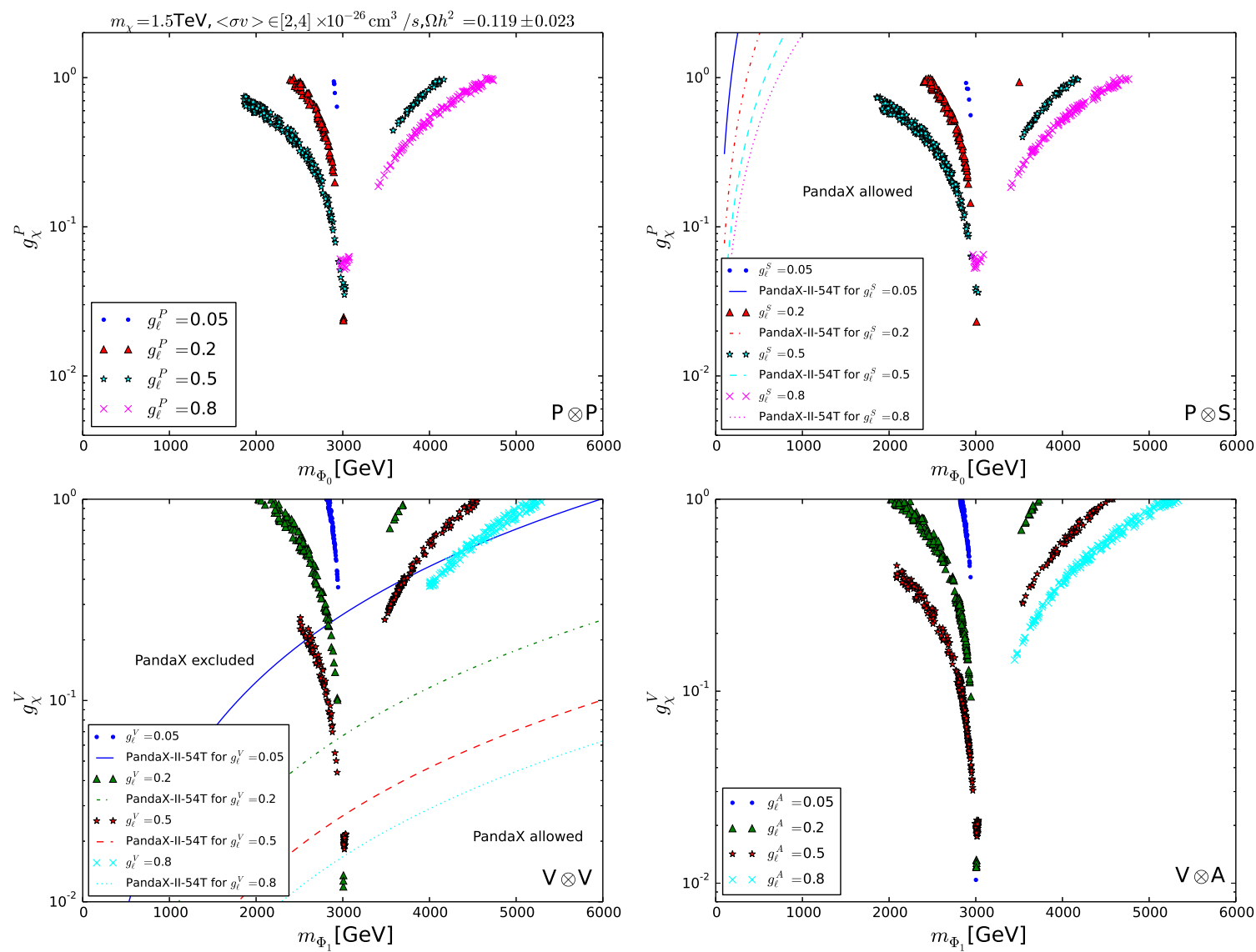

Figure 4. Scatter plots of the samples satisfying the DM relic density within $2 \sigma$ range of Planck observed value, LEP bound and the DAMPE excess, projected on the plane of $g_{\chi}$ versus $m_{\Phi}$ for $g_{\ell}=0.05,02,0.5,0.8$. All samples are required to produce averaged annihilation cross-section $\langle\sigma v\rangle$ today within $(2-4) \times 10^{-26} \mathrm{~cm}^{3} / \mathrm{s}$. The $90 \%$ C.L. exclusion limits from the current PandaX-II data is also shown [61].

the DM has no interactions with nucleus. The surviving samples for $V \otimes V$ coupling are largely excluded by the PandaX-II limits of DM-nucleus scattering. There are also limits from other direct detection experiments such as XENON1T [62] and LUX [63]. However, their current bounds are weaker than that of PandaX-II.

It should be mentioned that the vector mediator $\Phi_{1}$ can be produced at the LHC because of the loop-induced coupling between the mediator and light quarks, as shown in figure 5. The cross section in the narrow width limit is given by [64]

$$
\sigma_{p p \rightarrow l^{+} l^{-}}=\frac{\pi B R_{\Phi_{1 \rightarrow l^{+} l^{-}}}}{3 s} \sum_{q} C_{q \bar{q}}\left(m_{\Phi_{1}}^{2} / s\right)\left(g_{q}^{V^{2}}+g_{q}^{A^{2}}\right)
$$

where $B R_{\Phi_{1} \rightarrow l^{+} l^{-}}$is the branching ratio of the decay $\Phi_{1} \rightarrow l^{+} l^{-}$. The parton luminosity $C_{q \bar{q}}\left(m_{\Phi_{1}}^{2} / s\right)$ for the quark $q$ reads

$$
C_{q \bar{q}}(y)=\int_{y}^{1} d x \frac{f_{q}(x) f_{\bar{q}}(y / x)+f_{q}(y / x) f_{\bar{q}}(x)}{x},
$$




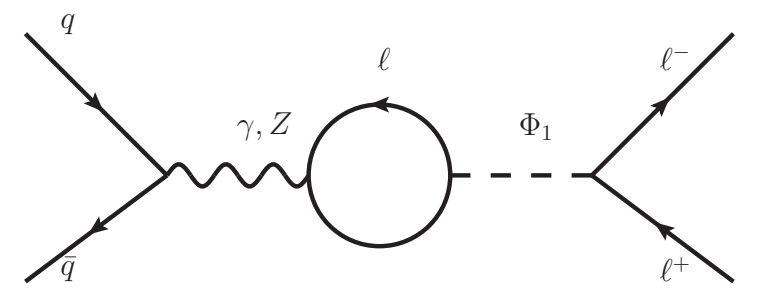

Figure 5. Feynman diagrams for the Drell-Yan process induced by a vector mediator $\Phi_{1}$ at the LHC.

\begin{tabular}{|l|c|c|c|}
\hline & $\left(g_{\chi}^{V}, g_{\ell}^{V}\right)=(0.21,0.05)$ & $\left(g_{\chi}^{V}, g_{\ell}^{V}\right)=(0.012,0.2)$ & $\left(g_{\chi}^{V}, g_{\ell}^{V}\right)=(0.2,0.5)$ \\
\hline$m_{\Phi_{1}}=3 \mathrm{TeV}$ & $5.2 \times 10^{-4}$ & $7.5 \times 10^{-3}$ & $5.0 \times 10^{-2}$ \\
\hline
\end{tabular}

Table 2. The cross section of dilepton production $p p \rightarrow \Phi_{1} \rightarrow \ell^{+} \ell^{-}$at $13 \mathrm{TeV}$ LHC, where the cross sections are in unit of fb. The benchmark points satisfy the DM relic density, the DAMPE $e^{+}+e^{-}$flux excess and the PandaX limits.

\begin{tabular}{|c|c|c|c|c|}
\hline$m_{\Phi}(\mathrm{TeV})$ & $\left(g_{\chi}^{V}, g_{\ell}^{V}\right)=(0.012,0.2)$ & $\left(g_{\chi}^{V}, g_{\ell}^{A}\right)=(0.6,0.2)$ & $\left(g_{\chi}^{P}, g_{\ell}^{P}\right)=(0.6,0.2)$ & $\left(g_{\chi}^{P}, g_{\ell}^{S}\right)=(0.6,0.2)$ \\
\hline 2.1 & - & $-4.27 \times 10^{-12}$ & $-2.31 \times 10^{-11}$ & $2.31 \times 10^{-11}$ \\
2.5 & - & $-2.78 \times 10^{-12}$ & $-1.65 \times 10^{-11}$ & $1.65 \times 10^{-11}$ \\
3.0 & $4.13 \times 10^{-13}$ & $-2 \times 10^{-12}$ & $-1.14 \times 10^{-11}$ & $1.14 \times 10^{-11}$ \\
3.6 & - & $-1.43 \times 10^{-12}$ & $-8.36 \times 10^{-12}$ & $8.36 \times 10^{-12}$ \\
\hline
\end{tabular}

Table 3. Same as table 2, but for the corrections to the anomalous magnetic moment of the muon $\Delta a_{\mu}$.

with $f_{q, \bar{q}}(x)$ being the quark and antiquark parton distribution function (PDF). We use MRST [65] to calculate the PDFs. The loop-induced couplings to quarks $g_{q}^{V}$ and $g_{q}^{A}$ are evaluated with package runDM [66]. The renormalization scale of the PDF and the couplings to quarks is set at $m_{\Phi_{1}}$. We choose some benchmark points that satisfy the DM relic density, the DAMPE $e^{+}+e^{-}$flux excess and the PandaX limits and calculate the corresponding cross section of the dilepton process $p p \rightarrow \Phi_{1} \rightarrow \ell^{+} \ell^{-}$at the $13 \mathrm{TeV}$ LHC, as given in table 2. We note that they are much lower than the current LHC-13 TeV sensitivity [67]. We also evaluate the associated production processes $p p \rightarrow \ell^{+} \ell^{-} \Phi_{0,1}$ and find they are negligibly small.

In table 3, we give the corrections to the muon $g-2$ that arise from our leptophilic interactions [68]. It can be seen that the couplings $V \otimes V$ and $P \otimes S$ can produce a positive correction, which, however, is less than the value required by explaining the deviation of the muon $g-2$ from its experimental measurement.

\section{An $\mathrm{U}(1)_{X}$ realization}

An an example of realizations of LDM, we introduce a Dirac fermionic DM field $(\chi)$ by imposing a $Z_{2}$ symmetry, under which all SM matter particles are even while $\chi$ is odd. 


\begin{tabular}{|c|c|c|c|c|c|c|c|c|c|c|c|}
\hline & $Q_{L}^{a}$ & $U_{R}^{a}$ & $D_{R}^{a}$ & $L_{L}^{a}$ & $e_{R}$ & $\mu_{R}$ & $\tau_{R}$ & $S$ & $T$ & $F$ & $\chi$ \\
\hline $\mathrm{U}(1)_{X}$ & 0 & 0 & 0 & 1 & 1 & 1 & 1 & $Q_{S}^{X}$ & 0 & $Q_{F}^{X}$ & 0 \\
\hline $\mathrm{U}(1)^{\prime}$ & 0 & 0 & 0 & 0 & 0 & 0 & 1 & 0 & $Q_{T}^{\prime}$ & $Q_{F}^{\prime}$ & $Q_{\chi}^{\prime}$ \\
\hline
\end{tabular}

Table 4. The $\mathrm{U}(1)_{X}$ quantum number for SM matter contents with the generation index $a=$ $(1,2,3)$. The complex scalars $S$ and $T$ are introduced to break the $\mathrm{U}(1)_{X}$ and $\mathrm{U}(1)^{\prime}$ gauge symmetry, respectively. The Dirac fermion $F$ that has charges of $\mathrm{U}(1)$, is introduced to generate kinetic mixing between the two $\mathrm{U}(1)$ gauge bosons.

Besides, we add a new $\mathrm{U}(1)_{X}$ gauge interactions for leptons only, with the corresponding gauge quantum numbers shown in table 4 . The complex scalars $S$ and $T$ are introduced to break the $\mathrm{U}(1)_{X}$ and $\mathrm{U}(1)^{\prime}$ gauge symmetry, respectively.

Such an assignment will cause the $\mathrm{U}(1)_{X}$ to be anomalous if there are no additional chiral fermions that are charged under $\mathrm{U}(1)_{X}$ other than the SM matter contents. One possible way is to introduce new matter particles to cancel the anomaly. For example, we can add the fourth chiral-like family with non-trivial $\mathrm{U}(1)_{X}$ quantum number, which satisfies the anomaly cancelation condition

$$
\sum_{i}\left(3 n_{i}+m_{i}\right)+3 k+l=0,
$$

with $n_{i}, m_{i}, k, l$ being the $\mathrm{U}(1)_{X}$ quantum numbers for quarks $\left(n_{i}\right)$, leptons $\left(m_{i}\right)$ of the first three family and the fourth family quarks $(k)$ and leptons $(l)$, respectively, such as $l=-3 m$ with universal $m_{i} \equiv m$ for $e, \mu, \tau$ leptons and trivial quantum numbers for all quarks. The fourth family can be very heavy by mixing with heavy vector-like fermions and can be compatible with current collider constraints.

Since the DM direct detection experiments will give stringent constraints, we require that the Dirac fermion DM $\chi$ will not carry $\mathrm{U}(1)_{X}$ quantum number but will transform non-trivially under an additional $\mathrm{U}(1)^{\prime}$ gauge symmetry. Such $\mathrm{U}(1)^{\prime}$ gauge symmetry will be broken by additional complex scalar field $T$. The couplings between DM and lepton pairs will be induced through kinetic mixing between $\mathrm{U}(1)_{X}$ and $\mathrm{U}(1)^{\prime}$. Given the gauge interaction $\mathrm{U}(1)_{X}$ is universal for all kinds of leptons, we can anticipate that the decay products will lead to equal final states lepton species. This approach is similar to vectorportal DM scenario. Since the DM is vector-like, there will be no additional anomaly in the model. New scalar $T$ or vector-like fermion $F$, which transform non-trivially under both $\mathrm{U}(1)_{X}$ and $\mathrm{U}(1)^{\prime}$, will induce non-trivial mixing between the two new $\mathrm{U}(1)$ gauge symmetry through the following interactions,

$$
\begin{aligned}
\mathcal{L} \supseteq & \left|D_{\mu} S\right|^{2}+\left|D_{\mu} T\right|^{2}-m_{S}^{2}|S|^{2}-m_{T}^{2}|T|^{2}-\lambda_{1}|S|^{4}-\lambda_{2}|T|^{4} \\
& -\lambda_{3}|S|^{2}|T|^{2}+i \bar{F} \gamma^{\mu} D_{\mu} F-m_{F} \bar{F} F
\end{aligned}
$$

with

$$
\begin{aligned}
D_{\mu} F & =\left(\partial_{\mu}-i Q_{F}^{X} g_{X} A_{\mu}^{X}-i Q_{F}^{\prime} g^{\prime} A_{\mu}^{\prime}\right) F \\
D_{\mu} S & =\left(\partial_{\mu}-i Q_{S}^{X} g_{X} A_{\mu}^{X}\right) S \\
D_{\mu} T & =\left(\partial_{\mu}-i Q_{T}^{\prime} g^{\prime} A_{\mu}^{\prime}\right) T .
\end{aligned}
$$


As mentioned above, an odd $Z_{2}$ parity is imposed for the Dirac fermion $\chi$ to act as a viable DM candidate. The masses of the scalar $T$ are assumed to be heavier than the DM mass so that the DM will not annihilate into them. We should note that in the scalar potential, possible terms involving standard model Higgs fields $H$ as $\left(T^{\dagger} T\right)\left(H^{\dagger} H\right),\left(S^{\dagger} S\right)\left(H^{\dagger} H\right)$ etc could appear. Such terms could contribute to the DM direct detection at two loop level.

The kinetic mixing between two gauge bosons can be parameterized as

$$
\mathcal{L} \supseteq-\frac{1}{4} F_{\mu \nu} F^{\mu \nu}-\frac{1}{4} F_{\mu \nu}^{\prime} F^{\mu \nu \prime}-\frac{\epsilon}{2} F_{\mu \nu}^{\prime} F^{\mu \nu}-\frac{1}{2} m_{1}^{2} A_{\mu} A^{\mu}-\frac{1}{2} m_{2}^{2} A^{\mu \prime} A_{\mu \prime},
$$

with

$$
\epsilon=-\frac{g_{X} g^{\prime}}{12 \pi^{2}} Q_{F}^{X} Q_{F}^{\prime} \log \left(\frac{m_{F}^{2}}{\mu^{2}}\right),
$$

after integrating out heavy fermion loops, or

$$
\epsilon=\frac{g_{1} g_{2}}{48 \pi^{2}} Q_{F} Q_{F}^{\prime} \log \left(\frac{m_{S}^{2}}{\mu^{2}}\right)
$$

after integrating out possible heavy scalar loops.

The matrix to remove the mixing is given as

$$
\left(\begin{array}{c}
\tilde{A}_{\mu} \\
\tilde{A}_{\mu}^{\prime}
\end{array}\right)=\left(\begin{array}{cc}
\frac{1}{\sqrt{1+\epsilon^{2}}} & 0 \\
-\frac{\epsilon}{\sqrt{1+\epsilon^{2}}} & 1
\end{array}\right)\left(\begin{array}{c}
A_{\mu} \\
A_{\mu}^{\prime}
\end{array}\right),
$$

with the Lagrangian involving the mass mixing

$$
\mathcal{L}=-\frac{1}{4} \tilde{F}_{\mu \nu} \tilde{F}^{\mu \nu}-\frac{1}{4} \tilde{F}_{\mu \nu}^{\prime} \tilde{F}^{\mu \nu \prime}-\frac{1}{2} m_{1}^{2} \tilde{A}_{\mu} \tilde{A}^{\mu}-\frac{1}{2} m_{2}^{2} \tilde{A}_{\mu}^{\prime} \tilde{A}^{\mu \prime}-m_{1}^{2} \epsilon \tilde{A}_{\mu} \tilde{A}^{\mu \prime} .
$$

Assuming identical masses for the scalars $m_{1}^{2}=m_{2}^{2}$, we obtain

$$
\left(\bar{\chi} \gamma^{\mu} \chi\right)\left(\bar{L} \gamma^{\nu} L\right)\left[\epsilon / m_{2}^{2}\right]
$$

To explain the DAMPE excess without conflicting with direct detection experiments, we can choose $m_{2} \simeq 3 \mathrm{TeV}$ and the mixing parameter $\epsilon \approx 1.0 \times 10^{-2}$. Such values can be obtained by requiring $g_{1}=g_{2} \approx 0.3$ with $Q_{1}=Q_{2}=1$.

\section{Conclusion}

In this work, we explained the recent DAMPE cosmic $e^{+}+e^{-}$excess in simplified leptophilic Dirac fermion dark matter (LDM) framework with a scalar $\left(\Phi_{0}\right)$ or vector $\left(\Phi_{1}\right)$ mediator. We found that the couplings $P \otimes S, P \otimes P, V \otimes A$ and $V \otimes V$ can fit the DAMPE data under the constraints from gamma-rays and cosmic-rays. However, for the $V \otimes V$ coupling, due to the stringent constraints from the PandaX-II data, the surviving samples only exist in the resonance region, $m_{\Phi_{1}} \simeq 2 m_{\chi}$. But for other couplings, the direct detection bounds can easily be evaded. We also studied the possible collider signatures of LDM, such as the Drell-Yan process $p p \rightarrow \Phi_{1} \rightarrow \ell^{+} \ell^{-}$, and the muon $g-2$. In the end, we constructed an $\mathrm{U}(1)$ extension of the SM to realize our simplified LDM model. 


\section{Acknowledgments}

G. Duan was supported by a visitor program of Nanjing Normal University, during which this work was finished. This work was supported by the National Natural Science Foundation of China (NNSFC) under grant No. 11705093, 11675242 and 11773075 , by the CAS Center for Excellence in Particle Physics (CCEPP), by the CAS Key Research Program of Frontier Sciences and by a Key R\&D Program of Ministry of Science and Technology of China under number 2017YFA0402200-04. FL is also supported by the Youth Innovation Promotion Association of Chinese Academy of Sciences (No. 2016288).

Open Access. This article is distributed under the terms of the Creative Commons Attribution License (CC-BY 4.0), which permits any use, distribution and reproduction in any medium, provided the original author(s) and source are credited.

\section{References}

[1] J. Chang, Dark Matter Particle Explorer: The First Chinese Cosmic Ray and Hard $\gamma$-ray Detector in Space, Chin. J. Space. Sci. 34 (2014) 550.

[2] DAMPE collaboration, J. Chang et al., The DArk Matter Particle Explorer mission, Astropart. Phys. 95 (2017) 6 [arXiv:1706.08453] [INSPIRE].

[3] DAMPE collaboration, G. Ambrosi et al., Direct detection of a break in the teraelectronvolt cosmic-ray spectrum of electrons and positrons, Nature 552 (2017) 63 [arXiv:1711.10981] [INSPIRE].

[4] Q. Yuan et al., Interpretations of the DAMPE electron data, arXiv:1711.10989 [INSPIRE].

[5] K. Fang, X.-J. Bi and P.-F. Yin, Explanation of the knee-like feature in the DAMPE cosmic $e^{-}+e^{+}$energy spectrum, arXiv:1711.10996 [INSPIRE].

[6] Y.-Z. Fan, W.-C. Huang, M. Spinrath, Y.-L.S. Tsai and Q. Yuan, A model explaining neutrino masses and the DAMPE cosmic ray electron excess, arXiv:1711.10995 [INSPIRE].

[7] P.-H. Gu and X.-G. He, Electrophilic dark matter with dark photon: from DAMPE to direct detection, Phys. Lett. B 778 (2018) 292 [arXiv:1711.11000] [INSPIRE].

[8] S. Chang, R. Edezhath, J. Hutchinson and M. Luty, Leptophilic Effective WIMPs, Phys. Rev. D 90 (2014) 015011 [arXiv:1402.7358] [INSPIRE].

[9] D. Schmidt, T. Schwetz and T. Toma, Direct Detection of Leptophilic Dark Matter in a Model with Radiative Neutrino Masses, Phys. Rev. D 85 (2012) 073009 [arXiv:1201.0906] [INSPIRE].

[10] P. Agrawal, S. Blanchet, Z. Chacko and C. Kilic, Flavored Dark Matter and Its Implications for Direct Detection and Colliders, Phys. Rev. D 86 (2012) 055002 [arXiv:1109.3516] [INSPIRE].

[11] C.D. Carone and R. Primulando, A Froggatt-Nielsen Model for Leptophilic Scalar Dark Matter Decay, Phys. Rev. D 84 (2011) 035002 [arXiv:1105.4635] [INSPIRE].

[12] P. Ko and Y. Omura, Supersymmetric $\mathrm{U}(1)_{B} \times \mathrm{U}(1)_{L}$ model with leptophilic and leptophobic cold dark matters, Phys. Lett. B 701 (2011) 363 [arXiv: 1012.4679] [INSPIRE].

[13] N. Haba, Y. Kajiyama, S. Matsumoto, H. Okada and K. Yoshioka, Universally Leptophilic Dark Matter From Non-Abelian Discrete Symmetry, Phys. Lett. B 695 (2011) 476 [arXiv: 1008.4777] [INSPIRE]. 
[14] Y. Farzan, S. Pascoli and M.A. Schmidt, AMEND: A model explaining neutrino masses and dark matter testable at the LHC and MEG, JHEP 10 (2010) 111 [arXiv:1005.5323] [INSPIRE].

[15] E.J. Chun, J.-C. Park and S. Scopel, Dirac gaugino as leptophilic dark matter, JCAP 02 (2010) 015 [arXiv:0911.5273] [INSPIRE].

[16] T. Cohen and K.M. Zurek, Leptophilic Dark Matter from the Lepton Asymmetry, Phys. Rev. Lett. 104 (2010) 101301 [arXiv:0909. 2035] [INSPIRE].

[17] H. Davoudiasl, Dark Matter with Time-Varying Leptophilic Couplings, Phys. Rev. D 80 (2009) 043502 [arXiv:0904.3103] [INSPIRE].

[18] A. Ibarra, A. Ringwald, D. Tran and C. Weniger, Cosmic Rays from Leptophilic Dark Matter Decay via Kinetic Mixing, JCAP 08 (2009) 017 [arXiv:0903.3625] [INSPIRE].

[19] B. Kyae, PAMELA/ATIC anomaly from the meta-stable extra dark matter component and the leptophilic Yukawa interaction, JCAP 07 (2009) 028 [arXiv:0902.0071] [INSPIRE].

[20] C.-R. Chen and F. Takahashi, Cosmic rays from Leptonic Dark Matter, JCAP 02 (2009) 004 [arXiv: 0810 .4110] [InSPIRE].

[21] E.A. Baltz and L. Bergstrom, Detection of leptonic dark matter, Phys. Rev. D 67 (2003) 043516 [hep-ph/0211325] [INSPIRE].

[22] Y. Bai and J. Berger, Lepton Portal Dark Matter, JHEP 08 (2014) 153 [arXiv:1402.6696] [INSPIRE].

[23] P. Schwaller, T.M.P. Tait and R. Vega-Morales, Dark Matter and Vectorlike Leptons from Gauged Lepton Number, Phys. Rev. D 88 (2013) 035001 [arXiv:1305.1108] [InSPIRE].

[24] L. Basso, O. Fischer and J.J. van der Bij, Natural Z' model with an inverse seesaw mechanism and leptonic dark matter, Phys. Rev. D 87 (2013) 035015 [arXiv:1207.3250] [INSPIRE].

[25] C.D. Carone, A. Cukierman and R. Primulando, On the Cosmic-Ray Spectra of Three-Body Lepton-Flavor-Violating Dark Matter Decays, Phys. Lett. B 704 (2011) 541 [arXiv: 1108.2084] [INSPIRE].

[26] W. Chao, Pure Leptonic Gauge Symmetry, Neutrino Masses and Dark Matter, Phys. Lett. B 695 (2011) 157 [arXiv: 1005.1024] [INSPIRE].

[27] S. Khalil, H.-S. Lee and E. Ma, Generalized Lepton Number and Dark Left-Right Gauge Model, Phys. Rev. D 79 (2009) 041701 [arXiv:0901.0981] [INSPIRE].

[28] Q.-H. Cao, E. Ma and G. Shaughnessy, Dark Matter: The Leptonic Connection, Phys. Lett. B 673 (2009) 152 [arXiv:0901.1334] [INSPIRE].

[29] A. Freitas and S. Westhoff, Leptophilic Dark Matter in Lepton Interactions at LEP and ILC, JHEP 10 (2014) 116 [arXiv: 1408.1959] [INSPIRE].

[30] N.F. Bell, Y. Cai, R.K. Leane and A.D. Medina, Leptophilic dark matter with $Z^{\prime}$ interactions, Phys. Rev. D 90 (2014) 035027 [arXiv:1407.3001] [INSPIRE].

[31] M.-C. Chen, J. Huang and V. Takhistov, Beyond Minimal Lepton Flavored Dark Matter, JHEP 02 (2016) 060 [arXiv: 1510.04694] [INSPIRE].

[32] J. Kile, A. Kobach and A. Soni, Lepton-Flavored Dark Matter, Phys. Lett. B 744 (2015) 330 [arXiv: 1411.1407] [INSPIRE].

[33] J. Kopp, L. Michaels and J. Smirnov, Loopy Constraints on Leptophilic Dark Matter and Internal Bremsstrahlung, JCAP 04 (2014) 022 [arXiv:1401.6457] [INSPIRE]. 
[34] K. Belotsky, M. Khlopov, C. Kouvaris and M. Laletin, Decaying Dark Atom constituents and cosmic positron excess, Adv. High Energy Phys. 2014 (2014) 214258 [arXiv:1403.1212] [INSPIRE].

[35] P.S.B. Dev, D.K. Ghosh, N. Okada and I. Saha, Neutrino Mass and Dark Matter in light of recent AMS-02 results, Phys. Rev. D 89 (2014) 095001 [arXiv: 1307.6204] [INSPIRE].

[36] A. Alves, A. Berlin, S. Profumo and F.S. Queiroz, Dark Matter Complementarity and the Z' Portal, Phys. Rev. D 92 (2015) 083004 [arXiv:1501.03490] [InSPIRE].

[37] S.M. Boucenna et al., Decaying Leptophilic Dark Matter at IceCube, JCAP 12 (2015) 055 [arXiv: 1507.01000] [INSPIRE].

[38] A. Berlin, D. Hooper and S.D. McDermott, Simplified Dark Matter Models for the Galactic Center Gamma-Ray Excess, Phys. Rev. D 89 (2014) 115022 [arXiv:1404.0022] [INSPIRE].

[39] S. Dutta, D. Sachdeva and B. Rawat, Signals of Leptophilic Dark Matter at the ILC, Eur. Phys. J. C 77 (2017) 639 [arXiv:1704.03994] [inSPIRE].

[40] M. Das and S. Mohanty, Leptophilic dark matter in gauged $L_{\mu}-L_{\tau}$ extension of MSSM, Phys. Rev. D 89 (2014) 025004 [arXiv:1306.4505] [InSPIRE].

[41] P.J. Fox and E. Poppitz, Leptophilic Dark Matter, Phys. Rev. D 79 (2009) 083528 [arXiv:0811.0399] [INSPIRE].

[42] X.-J. Bi, X.-G. He and Q. Yuan, Parameters in a class of leptophilic models from PAMELA, ATIC and FERMI, Phys. Lett. B 678 (2009) 168 [arXiv:0903.0122] [INSPIRE].

[43] A. Hamze, C. Kilic, J. Koeller, C. Trendafilova and J.-H. Yu, Lepton-Flavored Asymmetric Dark Matter and Interference in Direct Detection, Phys. Rev. D 91 (2015) 035009 [arXiv: 1410.3030] [INSPIRE].

[44] C.-J. Lee and J. Tandean, Lepton-Flavored Scalar Dark Matter with Minimal Flavor Violation, JHEP 04 (2015) 174 [arXiv:1410.6803] [INSPIRE].

[45] S. Baek and P. Ko, Phenomenology of $\mathrm{U}(1)_{L_{\mu}-L_{\tau}}$ charged dark matter at PAMELA and colliders, JCAP 10 (2009) 011 [arXiv:0811.1646] [INSPIRE].

[46] F. del Aguila, M. Chala, J. Santiago and Y. Yamamoto, Collider limits on leptophilic interactions, JHEP 03 (2015) 059 [arXiv:1411.7394] [INSPIRE].

[47] B. Fornal, Y. Shirman, T.M.P. Tait and J.R. West, Asymmetric dark matter and baryogenesis from $\mathrm{SU}(2)_{\ell}$, Phys. Rev. D 96 (2017) 035001 [arXiv:1703.00199] [InSPIRE].

[48] B. Fornal, Dark Matter and Baryogenesis from Non-Abelian Gauged Lepton Number, Mod. Phys. Lett. A 32 (2017) 1730018 [arXiv:1705.07297] [INSPIRE].

[49] J. Kile, Flavored Dark Matter: A Review, Mod. Phys. Lett. A 28 (2013) 1330031 [arXiv: 1308.0584] [INSPIRE].

[50] J. Kopp, V. Niro, T. Schwetz and J. Zupan, DAMA/LIBRA and leptonically interacting Dark Matter, Phys. Rev. D 80 (2009) 083502 [arXiv:0907.3159] [INSPIRE].

[51] A.W. Strong and I.V. Moskalenko, Propagation of cosmic-ray nucleons in the galaxy, Astrophys. J. 509 (1998) 212 [astro-ph/9807150] [INSPIRE].

[52] C. Evoli, D. Gaggero, D. Grasso and L. Maccione, Cosmic-Ray Nuclei, Antiprotons and Gamma-rays in the Galaxy: a New Diffusion Model, JCAP 10 (2008) 018 [arXiv: 0807.4730] [INSPIRE].

[53] A.M. Atoian, F.A. Aharonian and H.J. Volk, Electrons and positrons in the galactic cosmic rays, Phys. Rev. D 52 (1995) 3265 [InSPIRE]. 
[54] X. Huang, Y.-L.S. Tsai and Q. Yuan, LikeDM: likelihood calculator of dark matter detection, Comput. Phys. Commun. 213 (2017) 252 [arXiv:1603.07119] [InSPIRE].

[55] L. Zu, C. Zhang, L. Feng, Q. Yuan and Y.-Z. Fan, Constraints on box-shaped cosmic ray electron feature from dark matter annihilation with the AMS-02 and DAMPE data, arXiv:1711.11052 [INSPIRE].

[56] A. Alloul, N.D. Christensen, C. Degrande, C. Duhr and B. Fuks, FeynRules 2.0 - A complete toolbox for tree-level phenomenology, Comput. Phys. Commun. 185 (2014) 2250 [arXiv: 1310.1921] [INSPIRE].

[57] G. Bélanger et al., Indirect search for dark matter with MicrOMEGAs2.4, Comput. Phys. Commun. 182 (2011) 842 [arXiv: 1004.1092] [INSPIRE].

[58] DELPhi, OPAL, LEP Electroweak, ALEPH and L3 collaborations, S. Schael et al., Electroweak Measurements in Electron-Positron Collisions at W-Boson-Pair Energies at LEP, Phys. Rept. 532 (2013) 119 [arXiv:1302.3415] [INSPIRE].

[59] OPAL collaboration, G. Abbiendi et al., Tests of the standard model and constraints on new physics from measurements of fermion pair production at 189-GeV at LEP, Eur. Phys. J. C 13 (2000) 553 [hep-ex/9908008] [INSPIRE].

[60] A. Freitas, J. Lykken, S. Kell and S. Westhoff, Testing the Muon g-2 Anomaly at the LHC, JHEP 05 (2014) 145 [Erratum ibid. 09 (2014) 155] [arXiv:1402.7065] [INSPIRE].

[61] PandaX-II collaboration, X. Cui et al., Dark Matter Results From 54-Ton-Day Exposure of PandaX-II Experiment, Phys. Rev. Lett. 119 (2017) 181302 [arXiv:1708.06917] [InSPIRE].

[62] XENON collaboration, E. Aprile et al., First Dark Matter Search Results from the XENON1T Experiment, Phys. Rev. Lett. 119 (2017) 181301 [arXiv:1705.06655] [INSPIRE].

[63] LUX collaboration, D.S. Akerib et al., Results from a search for dark matter in the complete LUX exposure, Phys. Rev. Lett. 118 (2017) 021303 [arXiv: 1608. 07648] [INSPIRE].

[64] F. D'Eramo, B.J. Kavanagh and P. Panci, Probing Leptophilic Dark Sectors with Hadronic Processes, Phys. Lett. B 771 (2017) 339 [arXiv:1702.00016] [INSPIRE].

[65] A.D. Martin, W.J. Stirling, R.S. Thorne and G. Watt, Update of parton distributions at NNLO, Phys. Lett. B 652 (2007) 292 [arXiv:0706. 0459] [inSPIRE].

[66] F. D'Eramo, B.J. Kavanagh and P. Panci, You can hide but you have to run: direct detection with vector mediators, JHEP 08 (2016) 111 [arXiv:1605.04917] [INSPIRE].

[67] ATLAS collaboration, Search for new high-mass phenomena in the dilepton final state using $36 \mathrm{fb}^{-1}$ of proton-proton collision data at $\sqrt{\mathrm{s}}=13 \mathrm{TeV}$ with the ATLAS detector, JHEP 10 (2017) 182 [arXiv: 1707.02424] [INSPIRE].

[68] P. Agrawal, Z. Chacko and C.B. Verhaaren, Leptophilic Dark Matter and the Anomalous Magnetic Moment of the Muon, JHEP 08 (2014) 147 [arXiv: 1402.7369] [INSPIRE]. 